\title{
Diabetes mellitus among young adults in Sri Lanka-role of GAD antibodies in classification and treatment: The Sri Lanka Young Diabetes study
}

\author{
P. Katulanda • B. Shine - G. W. Katulanda - A. Silva • \\ E. L. Asfir • R. Sheriff • N. Somasundaram • \\ A. E. Long • P. J. Bingley • M. I. McCarthy • A. Clark • \\ D. R. Matthews
}

Received: 9 April 2008 / Accepted: 29 April 2008 / Published online: 5 June 2008

(C) Springer-Verlag 2008

\begin{abstract}
Aims/hypothesis Diabetes mellitus is increasing among young adult South Asians. The aim of this study was to determine the prevalence and phenotypic characteristics of diabetes subtypes based on GAD65 autoantibody (GADA) status in those with young adult-onset diabetes in Sri Lanka. Methods Clinical, metabolic and GADA data were available for 992 consecutively recruited individuals with diabetes aged $\leq 45$ years (age at diagnosis $16-40$ years).
\end{abstract}

Electronic supplementary material The online version of this article (doi:10.1007/s00125-008-1052-6) contains supplementary material, which is available to authorised users.

P. Katulanda $(\bowtie) \cdot$ M. I. McCarthy $\cdot$ A. Clark $\cdot$ D. R. Matthews Oxford Centre for Diabetes, Endocrinology and Metabolism, Churchill Hospital,

Old Road, Headington,

Oxford OX3 7LJ, UK

e-mail: prasad.katulanda@ocdem.ox.ac.uk

P. Katulanda $\cdot$ A. Silva $\cdot$ E. L. Asfir $\cdot$ R. Sheriff

Diabetes Research Unit, Department of Clinical Medicine,

University of Colombo,

Colombo, Sri Lanka

B. Shine $\cdot$ G. W. Katulanda

Department of Clinical Biochemistry, John Radcliffe Hospital,

Oxford, UK

N. Somasundaram

National Hospital of Sri Lanka,

Colombo, Sri Lanka

A. E. Long · P. J. Bingley

Department of Clinical Science at North Bristol, University of Bristol,

Bristol, UK
Participants were classified according to the following definitions: type 1 diabetes, insulin-dependent $<6$ months from diagnosis; latent autoimmune diabetes in adults (LADA), GADA-positive, age $\geq 30$ years and insulin-independent $\geq 6$ months from diagnosis; type 2 diabetes, GADA-negative and insulin-independent $\geq 6$ months from diagnosis.

Results The median (interquartile range) age at diagnosis and diabetes duration were 33.0 (29.0-36.1) and 4.0 (1.1$7.1)$ years, respectively; $42.1 \%$ were male. GADA positivity was seen in $5.4 \%$ of participants $(n=54)$ and GADA levels negatively correlated with age at diagnosis $(p<0.0001)$, BMI $(p<0.0001)$ and time to insulin requirement $(p=0.006)$. Type 1 diabetes, type 2 diabetes and LADA were present in $7.0 \%, 89.7 \%$ and $2.6 \%$, respectively. The remaining $0.7 \%$ of the participants were GADA-positive, insulin independent $\geq 6$ months from diagnosis and were diagnosed at age $<30$ years. The metabolic syndrome and homeostasis model assessment of beta cell function (HOMA \%B) were lowest in GADA-positive type 1 diabetes and increased progressively in latent autoimmune diabetes, GADA-negative type 1 diabetes and type 2 diabetes. Among those requiring insulin, $69.2 \%$ had fasting C-peptide levels in the lowest quartile, whereas only $19.5 \%$ were GADA-positive $(p<0.0001)$.

Conclusions/interpretation The prevalence of GADApositive autoimmune diabetes is low among individuals with young adult-onset diabetes in Sri Lanka. Young-onset diabetic phenotypes appear as a continuum from autoimmune type 1 diabetes to type 2 diabetes.

Keywords Autoimmune - GAD65 autoantibodies · Insulin . LADA · South Asian · Sri Lankans · Type 1 diabetes .

Type 2 diabetes · Young adults 


\begin{tabular}{|c|c|}
\hline \multicolumn{2}{|c|}{ Abbreviations } \\
\hline GADA & GAD65 autoantibodies \\
\hline HOMA \%B & $\begin{array}{l}\text { homeostasis model assessment of beta cell } \\
\text { function }\end{array}$ \\
\hline IA-2A & islet antigen- 2 autoantibodies \\
\hline ICA & islet cell autoantibodies \\
\hline IQR & interquartile range \\
\hline LADA & latent autoimmune diabetes in adults \\
\hline OHA & oral hypoglycaemic agents \\
\hline UKPDS & United Kingdom Prospective Diabetes Study \\
\hline
\end{tabular}

\section{Introduction}

Diabetes mellitus is increasingly diagnosed among young adults worldwide [1]. The aetiological and phenotypic heterogeneity of diabetes in this age group poses diagnostic and therapeutic challenges to clinicians $[2,3]$. The clinical spectrum of diabetes has not been adequately characterised among young adult South Asians, amongst whom diabetes has emerged as an important public health problem [4-6].

The recent international classifications have recognised the importance of the pathophysiological basis of different subtypes of diabetes [7, 8]. Type 1 diabetes, caused by autoimmune destruction of the pancreatic beta cells, leads to early insulin dependency and is characterised by the presence of autoantibodies to islet cell antigens (GAD65 [GADA], islet cell cytoplasm [ICA] and islet antigen-2 [IA2A]) [7]. Type 2 diabetes results from a combination of a deficiency in insulin secretion and insulin resistance and is not typically associated with islet autoantibodies; those affected can be initially managed by a combination of lifestyle measures and oral therapy. GADA and other islet autoantibodies are present in a subset of adult individuals presenting clinically with type 2 diabetes [9]. Because of the slowly progressive nature of this subtype of diabetes to insulin dependency, it was called latent autoimmune diabetes in adults (LADA) $[10,11]$. An age limit of $\geq 30$ years has been proposed to define LADA by the Immunology of Diabetes Society [12]. However, islet antibody-positive and slowly progressive diabetes has also been described in patients younger than 30 years of age [13].

In a large cohort of white European individuals $(n=3,672)$ aged $25-65$ years in the United Kingdom Prospective Diabetes Study (UKPDS) the prevalence of LADA was $10 \%$ [14]. There is paucity of information on autoimmune diabetes among South Asians. The available studies with relatively small sample sizes have produced conflicting data. In a study by Pan et al. on 269 young South Indians, the prevalence of GADA positivity was reported as $9.0 \%$ [15]. A high prevalence of $27.0 \%$ was reported by Tica et al. $(n=33)$ [16], while Britten et al. $(n=$ 500) [17] and Sahu et al. $(n=96)$ [18] reported prevalences of $1.6 \%$ and $3.0 \%$, respectively.

The aims of this study were to determine the prevalence of different subtypes of diabetes mellitus based on GADA positivity and insulin dependency among South Asians with young adult-onset (16-40 years) diabetes in Sri Lanka and to compare the phenotypic characteristics among the GADA-negative and positive patients. We also examined the association of GADA and fasting C-peptide with requirement for insulin.

\section{Methods}

Participants In this multicentre study, a consecutive sample of 1,007 individuals, who had been diagnosed between 16 and 40 years of age and were $\leq 45$ years at the time of the study, were recruited from the three largest hospitals (National Hospital of Sri Lanka, Colombo South Teaching Hospital and Colombo North Teaching Hospital), diabetes and medical outpatient clinics of selected government hospitals, referrals from physicians or general practitioners and the National Diabetes Centre in Sri Lanka, between June 2005 and February 2006. At these centres the total number of diabetic patient visits of all ages exceeded 20000 per month. During the recruitment period, 1,214 eligible individuals were invited; the response rate was $83 \%$. Pregnant women were not eligible. The study was conducted in accordance with the principles of the Declaration of Helsinki and was approved by the Ethical Review Committee of the University of Colombo. All participants provided informed written consent. This report is based on 992 participants after excluding nine with mitochondrial diabetes and six with incomplete data.

Data collection Recruited participants attended a research unit for data collection after an overnight fast. Blood samples for biochemical and immunological analysis were obtained prior to the morning insulin or oral therapy. Detailed demographic data, clinical and treatment history were recorded using an interviewer-administered questionnaire. The data were verified using medical records. Anthropometric measurements and blood pressure were recorded according to standard methods [19]. Body fat percentage was estimated using a bio-electrical impedance analysis technique (Bodystat 1500; Bodystat, Douglas, Isle of Man).

Laboratory methods Fasting plasma glucose was measured by a glucose oxidase method [20]. Total cholesterol, HDLcholesterol (by precipitation) and triacylglycerol were measured by an enzymatic colorimetric method. Glucose 
and lipid assays were performed using reagents supplied by Roche Diagnostics (Mannheim, Germany) in a Hitachi 704 chemical autoanalyser (Roche Diagnostics) at the Reproductive and Endocrine Laboratory in the University of Colombo. LDL-cholesterol was estimated using the Friedwald equation [21]. $\mathrm{HbA}_{1 \mathrm{c}}$ was measured by a HPLC technique (Biorad, Hercules, CA, USA) [22] at the National Diabetes Centre in Colombo. Samples for measurement of insulin, C-peptide and GADA were transported in dry ice to the UK. Fasting insulin and C-peptide measurements were made by a two-site sandwich immunoassay using a direct chemiluminescence method (Bayer Healthcare, Walpole, MA, USA).

GADA were measured by a radioimmunoassay using ${ }^{35}$ S-labelled full-length GAD65, and results were expressed in $\mathrm{WHO}$ units $/ \mathrm{ml}$ derived from a standard curve calibrated from the international reference material (National Institute for Biological Standards and Control code 97/550). Samples were considered positive if they had levels above 14 WHO units $/ \mathrm{ml}$ (the 97.5th percentile of 2860 school children) [23]. The inter-assay CV of the GADA assay was $13 \%$ at $14 \mathrm{WHO}$ units/ml. The GADA assay achieved a laboratory-defined sensitivity of $94 \%$ with $96 \%$ specificity in the fourth proficiency evaluation of the Antibody Standardization Program [24]. Beta cell function was estimated by homeostatic model assessment (HOMA \%B) [25].

Terms and classifications Those for whom insulin treatment was started within 6 months from diagnosis were classified as having type 1 diabetes, and these participants were further subclassified as having GADA-positive type 1 diabetes or GADA-negative type 1 diabetes. Those who were GADA-positive but insulin independent during the first 6 months from diagnosis were termed latent autoimmune diabetes irrespective of age. The participants with latent autoimmune diabetes aged $\geq 30$ years at diagnosis were considered to have LADA [12]. Participants who were GADA negative and insulin independent for equal to or more than 6 months from diagnosis were considered to have type 2 diabetes. The metabolic syndrome was diagnosed according to the International Diabetes Federation criteria [26].

Statistical methods Data were double entered, checked for consistency and analysed with SPSS version 14 (SPSS, Chicago, IL, USA). Values are shown as mean $\pm \mathrm{SD}$, median \pm interquartile range (IQR) or percentage with $95 \%$ CIs, according to the distribution of the data. The $\chi^{2}$ statistic, Mann-Whitney $U$ test, Kruskall-Wallis test and Pearson's correlation test were applied whenever appropriate. Multiple stepwise binary logistic regression was performed to assess the covariates for insulin therapy. A $p$ value of $<0.05$ was considered statistically significant.

\section{Results}

Characteristics of the study participants Among the 992 participants, $42.1 \%$ were men. The median values (IQR) for age, age at diagnosis and duration of diabetes (in years) were 38.0 (33.1-41.1), $33.0(29.0-36.1)$ and $4.0(1.1-7.1)$, respectively. The treatment patterns were lifestyle alone, $13.5 \%$; oral therapy alone, $70.5 \%$; and insulin, $16.0 \%$. The mean $\mathrm{HbA}_{1 \mathrm{c}}$ was $8.1 \pm 2.0 \%$; BMI, $24.7 \pm 4.0 \mathrm{~kg} / \mathrm{m}^{2}$; and waist circumference, $87.3 \pm 9.7 \mathrm{~cm}$. Eight individuals $(0.8 \%)$ had a history of pancreatic disease.

Prevalence of GADA positivity and subtypes of diabetes The prevalence of GADA-positive diabetes in this cohort was $5.4 \%(n=54 ; 95 \%$ CI $4.0-6.8)$. The prevalence of GADA positivity was much higher among those who were young and had a lower BMI compared with those who were older and more obese (Fig. 1). GADA positivity among men and women was $7.4 \%$ (95\% CI $4.9-9.9)$ and $4.0 \%(95 \%$ CI $2.4-5.6)$, respectively $(p=0.028)$. In this cohort, $7.0 \%(95 \%$ CI 5.4-8.6) were diagnosed with type 1 diabetes, $89.7 \%$ (95\% CI 87.8-91.6) with type 2 diabetes and $2.6 \%$ (95\% CI 1.6-3.6) with LADA. Seven $(0.7 \%$; $95 \%$ CI $0.2-1.2)$ patients who were diagnosed aged $<30$ years were GADA positive and insulin independent during the first 6 months from diagnosis. Type 1 diabetes was equally distributed between men $(8.6 \%, 95 \%$ CI $5.9-11.3)$ and women $(6.1 \%$, 95\% CI 4.1-8.1). Among those with type 1 diabetes, $29.6 \%$ (95\% CI 18.3-39.7) were GADA positive, which equates to $2.1 \%$ of the total sample. The prevalence of GADApositive type 1 diabetes was higher among men than women $(3.8 \%$ vs $0.9 \%, p=0.003)$.

Clinical features associated with GAD autoantibody positivity and insulin therapy In all 992 patients, GADA levels negatively correlated with age at diagnosis $(r=-0.224$, $p<0.0001)$, fasting C-peptide levels $(r=-0.241, p<0.0001)$, BMI $(r=-0.210, p<0.0001)$ and time to insulin treatment $(r=0.220, p=0.006)$ (Fig. 2). GADA positivity was seen in $19.5 \%$ of those who required insulin (time from diagnosis

a

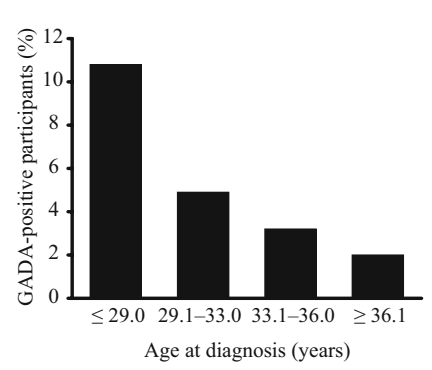

b

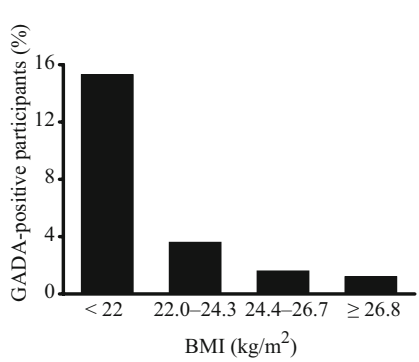

Fig. 1 GADA positivity according to quartiles of age at diagnosis (a) and BMI (b) among young adult-onset diabetes patients in Sri Lanka 
not taken into account) compared with $2.8 \%$ of those who did not require insulin $(p<0.0001)$. GADA positivity (corrected for age at diagnosis) correlated with insulin treatment $(r=0.213, p<0.0001)$.

Among those treated with insulin, $69.2 \%$ had fasting C-peptide levels in the lowest quartile and $19.5 \%$ were GADA positive $(p<0.0001)$. We examined the effect of values in the lowest quartile of fasting C-peptide, GADA positivity, age at diagnosis, BMI and waist circumference on insulin requirement by forward stepwise logistic regression analysis. Among the covariates, age at diagnosis, fasting C-peptide and GADA positivity remained significantly associated with insulin therapy. The strongest effect was seen with age at diagnosis $\leq 25$ years (OR 11.2, 95\% CI 5.9-21.3, $p=1.94 \times 10^{-13}$ ), followed by fasting C-peptide (OR 7.2, 95\% CI 4.8-10.9, $p=6.83 \times 10^{-21}$ ) and GADA positivity (OR $3.3,95 \%$ CI $1.6-6.8, p=0.002$ ).

Phenotypic characteristics among subtypes of diabetes The phenotypic characteristics of GADA-positive and GADAnegative participants are presented in Table 1. The comparison of phenotypic characteristics among individual subtypes is shown in Electronic supplementary material (ESM) Table 1. Significant differences were seen between GADA-negative and GADA-positive categories in all parameters examined, except LDL-cholesterol. Compared with those that tested negative for GADA, GADA-positive participants had been diagnosed at a younger age, were leaner, had a lower frequency of hypertension, presented
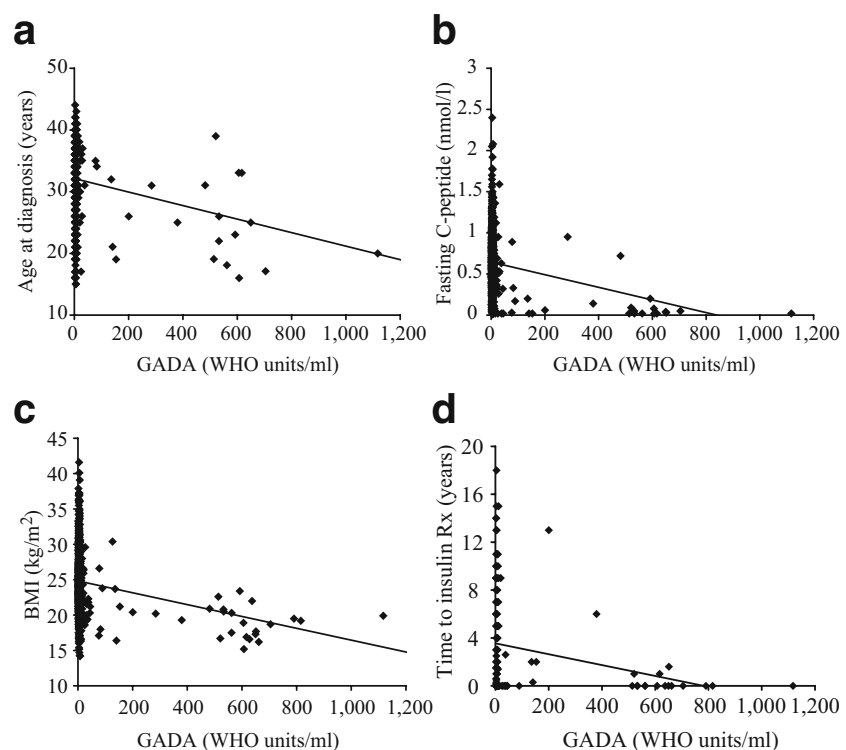

Fig. 2 Scatter plots illustrating the associations of GADA levels with age at diagnosis (a), fasting C-peptide level (b), BMI (c) and time from diagnosis to insulin treatment (d) among young adult-onset diabetic individuals in Sri Lanka. Rx, treatment more frequently with symptoms and commonly had a history suggestive of ketoacidosis. GADA-positive patients also had a lower prevalence of the metabolic syndrome and its components, lower markers of beta cell function (fasting C-peptide and HOMA \%B), less frequently had a family history of diabetes and had a lower prevalence of acanthosis nigricans. A gradual progression of values (from low to high, or vice versa) was observed for the metabolic syndrome, its components and the other phenotypic characteristics across the GADA-positive type 1 diabetes, latent autoimmune diabetes (GADA positive and insulin independent $\geq 6$ months from diagnosis, irrespective of age at diagnosis), GADA-negative type 1 diabetes and type 2 diabetes subgroups (ESM Table 1).

Treatment patterns and glycaemic outcome in patients with latent autoimmune diabetes Among those with latent autoimmune diabetes, $70 \%$ (23 out of 33) managed their condition by lifestyle modification or taking oral hypoglycaemic agents (OHA), and 35\% of this subgroup (eight out of 23) had good glycaemic control $\left(\mathrm{HbA}_{1 \mathrm{c}} \leq 7 \%\right)$ compared with only $20 \%$ (two out of ten) of the subgroup requiring insulin (ESM Table 2). Those with good glycaemic control while on lifestyle modification or OHA had higher beta cell function (median HOMA \%B 57.9, IQR 46.4-61.1), despite having a longer duration of diabetes (median 5.6 years, IQR 3.6-9.1).

\section{Discussion}

This is the largest multicentre study to date on young-onset diabetes in South Asians. The sample was recruited from hospitals and clinics in Sri Lanka, and the large size of this sample and the recruitment strategy enabled calculation of prevalence estimates for different diabetes subtypes and comparison of their phenotypic characteristics. It is likely that the prevalence of autoimmune diabetes was underestimated in this cross-sectional and post-diagnosis study as GADA might disappear post-diagnosis [27]. Furthermore, only GADA were tested, and participants, particularly those diagnosed with type 1 diabetes, may have had other types of islet autoantibodies. GADA persistence for $>6$ years post-diagnosis has been shown in LADA in the UKPDS and by Borg et al. in Sweden [28, 29]. Differences in assay techniques, units of measurement, cut-off values for GADA positivity and definitions of LADA also influence disparities in the data between different studies.

The prevalence of GADA positivity in this study (5.4\%) was lower than that reported by Pan et al. (9.0\%) for a younger cohort (mean age 31.2 years) of South Indians [15] and that reported by Kanungo and Sanjeevi (7\%) for 
Table 1 Clinical and metabolic characteristics of GADA-positive and GADA-negative individuals with young adult-onset diabetes in Sri Lanka

\begin{tabular}{|c|c|c|c|}
\hline Classification & GADA-positive diabetes & GADA-negative diabetes & $p$ value \\
\hline Number & 54 & 938 & \\
\hline Age at recruitment (years) & $31.6(24.1-39.1)$ & $38.1(34.0-41.1)$ & $<0.0001$ \\
\hline Age at diagnosis (years) & $26.5(21.1-33.0)$ & $33.0(29.0-36.1)$ & $<0.0001$ \\
\hline Duration of diabetes (years) & $4.0(1.1-6.1)$ & $4.0(1.1-7.1)$ & 0.708 \\
\hline $\operatorname{BMI}\left(\mathrm{kg} / \mathrm{m}^{2}\right)$ & $20.5(18.9-22.6)$ & $24.5(22.3-26.9)$ & $<0.0001$ \\
\hline Waist circumference $(\mathrm{cm})$ & $74.8(71.0-81.5)$ & $87.0(82.0-93.0)$ & $<0.0001$ \\
\hline WHR & $0.86(0.81-0.91)$ & $0.92(0.88-0.95)$ & $<0.0001$ \\
\hline Body fat $(\%)$ & $21.2(17.5-29.3)$ & $29.9(21.5-35.4)$ & $<0.0001$ \\
\hline HDL-cholesterol (mmol/l) & $1.1(0.9-1.5)$ & $1.0(0.9-1.2)$ & 0.001 \\
\hline LDL-cholesterol (mmol/1) & $3.2(2.7-3.8)$ & $3.2(2.6-3.8)$ & 0.865 \\
\hline Triacylglycerol (mmol/l) & $0.9(0.8-1.5)$ & $1.5(1.1-2.1)$ & $<0.0001$ \\
\hline $\mathrm{HbA}_{1 \mathrm{c}}(\%)$ & $9.2(7.1-11.4)$ & $7.8(6.4-9.2)$ & $<0.0001$ \\
\hline Fasting C-peptide (nmol/l) & $0.15(0.02-0.51)$ & $0.61(0.43-0.82)$ & $<0.0001$ \\
\hline HOMA B\% & $17.6(2.1-42.8)$ & $44.4(26.1-72.4)$ & $<0.0001$ \\
\hline Current insulin treatment & $57.4[44.2-70.6]$ & $13.6[11.4-15.8]$ & $<0.0001$ \\
\hline Definite or probable DKA & $25.9[14.2-37.6]$ & $7.2[5.5-8.9]$ & $<0.0001$ \\
\hline Hypertension & $7.4[0.4-14.4]$ & $33.0[30.0-36.0]$ & $<0.0001$ \\
\hline Family history of diabetes & $51.9[38.6-65.2]$ & $74.6[71.8-77.4]$ & $<0.0001$ \\
\hline Acanthosis nigricans & $7.4[0.4-14.4]$ & $21.6[19.0-24.2]$ & 0.020 \\
\hline Symptomatic presentation & $94.4[88.3-100.0]$ & $72.1[69.2-75.0]$ & 0.001 \\
\hline Metabolic syndrome & $18.5[8.1-28.9]$ & $62.5[59.4-65.6]$ & $<0.0001$ \\
\hline
\end{tabular}

Data are median (IQR) or percentage $[95 \% \mathrm{CI}]$. The $p$ values were calculated using the Mann-Whitney $U$ test, for continuous variables, or the $\chi^{2}$ test, for dichotomous variables

DKA, diabetic ketoacidosis

patients with type 2 diabetes from Cuttack in India [30]. The prevalence of LADA based on GADA (2.6\%) in our cohort is similar to that reported in two of the previous studies on South Asians; 3.0\% $(n=96)$ by Sahu et al. and $1.6 \%(n=500)$ by Britten et al. $[17,18]$, but is lower than the prevalence $(27 \%)$ reported in a different study $(n=33)$ [16]. The frequency of LADA among young adult South Asians in this study was much lower than that reported for young adults in the UKPDS (34\% and 14\% in those aged between 25-34 years and 35-44 years, respectively), the Botnia study ( $19.3 \%$ in those aged $\leq 45$ years) and in a Chinese population $(16.1 \%$ among a sample with a mean age at diagnosis of 40.6 years) [14, 31, 32]. A study from Sardinia on an older cohort with type 2 diabetes (3575 years) and a study from North America and Europe on drug-naive type 2 diabetic patients (30-75 years) have reported lower prevalences of GADA positivity $(5.1 \%$ and $4.2 \%$, respectively) than the UKPDS and the Botnia studies $[33,34]$. The higher ethnic predisposition to, and therefore higher prevalence of, type 2 diabetes among South Asians compared with Europeans and East Asians may contribute to the lower proportional prevalence of GADA positivity among patients with young adult-onset diabetes in Sri Lanka [35]. In addition, the possible lower genetic predisposition of South Asians to autoimmune diabetes compared with Europeans may play a role [36].
These data from this large sample of South Asians confirm the associations of GADA positivity with younger age at onset of diabetes and rapid progression to, and higher prevalence of, insulin requirement, as previously shown in European studies [14, 29]. Despite being statistically significant, the strength of the associations of GADA levels with age at diagnosis, fasting C-peptide, BMI and time to insulin treatment is low (Fig. 2). Phenotypic differences in anthropometry, prevalence of the metabolic syndrome and beta cell function in subtypes of diabetes in young South Asian adults were similar to those seen in populations from other countries [34, 37], except for BMI, which was lower for all subtypes of diabetes in the South Asians.

The proportionate prevalence of type 1 diabetes in our sample $(7.0 \%)$ is similar to that reported in 1000 Chinese, Malaysian and Indian individuals aged 12-40 years $(10.0 \%)$ [15]. In contrast, type 1 diabetes was reported in $61.4 \%$ of all new diabetic individuals aged 15-29 years from nine European countries between 1996 and 1997 [38]. GADA positivity among those with the type 1 phenotype in our cohort was $\sim 30 \%$. This is lower than the $40.0 \%$ among North Indian [39], 44\% among black African [40] and $69.0 \%$ of Japanese individuals [41] reported from similar cross-sectional studies, and the $78 \%$ among white Europeans aged 20-40 years reported by the Belgium diabetes registry [42]. The higher prevalence of type 1 diabetes 
among young adult male individuals has been previously reported for non-South Asians [38, 43, 44]. In this cohort from Sri Lanka, the prevalence of pancreatic diseases was lower than in reports from North India [45, 46].

Our phenotypic data on diabetes subtypes support the growing consensus that the clinical phenotypes of diabetes form a continuum from autoimmune type 1 diabetes to type 2 diabetes, with intermediate phenotypes, based on aetiology [47-49]. For definite aetiological diagnosis of every diabetic individual, all islet autoantibodies and a battery of genetic tests would be required. This would be extremely costly and prohibitive in clinical practice. Even if this were to be undertaken, heterogeneity within patient groups would exclude the application of uniform, group-based clinical management protocols. Randomised prospective studies are needed to determine the usefulness of complex islet cell antibody-based classification of diabetes subtypes on clinical outcomes.

The treatment of young-onset diabetes in Sri Lanka is not based on islet autoantibody status, and insulin requirement is assessed in response to treatment in the absence of ketoacidosis. Good glycaemic control and preserved beta cell function (median HOMA \%B 57.9) were present in $35 \%$ of patients with latent autoimmune diabetes treated with OHA or lifestyle modification (median diabetes duration 5.6 years). In the LADA subgroup of patients younger than 34 years in the UKPDS study, $84 \%$ required insulin within 6 years due to 'hyperglycaemic symptoms or when the fasting plasma glucose was above $15 \mathrm{mmol} / \mathrm{l}$ ' [14]. Among patients with latent autoimmune diabetes in our cohort (mean age 35 years), $48.5 \%(n=16)$ were either on insulin or had a fasting plasma glucose level $>15 \mathrm{mmol} / \mathrm{l}$ at a mean diabetes duration of 5.5 years. This could indicate that latent autoimmune diabetes progresses less rapidly in South Asians than in European individuals. The cross-sectional and retrospective data in this study showed that fasting C-peptide was a more sensitive indicator of insulin requirement than GADA positivity.

In summary, we report that autoimmune diabetes is less common in South Asian young adult-onset diabetes in Sri Lanka than in European populations. The higher proportional prevalence of type 2 diabetes provides evidence for the strong recommendation of effective primary preventive programmes targeting lifestyle modification among South Asians [50].

Acknowledgements The authors are grateful to J. Boldison, S. Rokni and K. Chandler of the University of Bristol for assistance with the GADA assay. The National Diabetes Centre Sri Lanka, the Endocrine and Reproductive Laboratory of the University of Colombo and the Department of Clinical Biochemistry of the John Radcliffe Hospital are acknowledged for providing support with respect to the chemical assays. The Sri Lanka Young Diabetes Study group (http:// www.ocdem.com/index.php?option $=$ com_content\&task=view \& $\mathrm{id}=145 \&$ Itemid=147, accessed 6 May 2008) is acknowledged for referring patients to the study, data collection work and for logistical support.

Duality of interest The authors declare that there is no duality of interest associated with this manuscript. Funding of the NIHR Biomedical Research Centre Programme is acknowledged. P. Katulanda is a Commonwealth Postgraduate Scholar.

\section{References}

1. Alberti G, Zimmet P, Shaw J, Bloomgarden Z, Kaufman F, Silink M (2004) Type 2 diabetes in the young: the evolving epidemic: the International Diabetes Federation consensus workshop. Diabetes Care 27:1798-1811

2. Chan JC, Ng MC (2003) Lessons learned from young-onset diabetes in China. Curr Diab Rep 3:101-107

3. Owen KR, Shepherd M, Stride A, Ellard S, Hattersley AT (2002) Heterogeneity in young adult onset diabetes: aetiology alters clinical characteristics. Diabet Med 19:758-761

4. Katulanda P, Sheriff MH, Matthews DR (2006) The diabetes epidemic in Sri Lanka - a growing problem. Ceylon Med J 51:26-28

5. Mohan V, Deepa M, Deepa R et al (2006) Secular trends in the prevalence of diabetes and impaired glucose tolerance in urban South India-the Chennai Urban Rural Epidemiology Study (CURES-17). Diabetologia 49:1175-1178

6. Ramachandran A, Snehalatha C, Baskar AD et al (2004) Temporal changes in prevalence of diabetes and impaired glucose tolerance associated with lifestyle transition occurring in the rural population in India. Diabetologia 47:860-865

7. American Diabetes Association (2003) Report of the expert committee on the diagnosis and classification of diabetes mellitus. Diabetes Care 26(Suppl 1):S5-S20

8. Alberti KG, Zimmet PZ (1998) Definition, diagnosis and classification of diabetes mellitus and its complications. Part 1: diagnosis and classification of diabetes mellitus provisional report of a WHO consultation. Diabet Med 15:539-553

9. Groop LC, Bottazzo GF, Doniach D (1986) Islet cell antibodies identify latent type I diabetes in patients aged 35-75 years at diagnosis. Diabetes 35:237-241

10. Tuomi T, Groop LC, Zimmet PZ, Rowley MJ, Knowles W, Mackay IR (1993) Antibodies to glutamic acid decarboxylase reveal latent autoimmune diabetes mellitus in adults with a noninsulin-dependent onset of disease. Diabetes 42:359-362

11. Zimmet PZ, Tuomi T, Mackay IR et al (1994) Latent autoimmune diabetes mellitus in adults (LADA): the role of antibodies to glutamic acid decarboxylase in diagnosis and prediction of insulin dependency. Diabet Med 11:299-303

12. Palmer JP, Hampe CS, Chiu H, Goel A, Brooks-Worrell BM (2005) Is latent autoimmune diabetes in adults distinct from type 1 diabetes or just type 1 diabetes at an older age? Diabetes 54(Suppl 2): S62-S67

13. Lohmann T, Nietzschmann U, Kiess W (2000) 'Lady-like': is there a latent autoimmune diabetes in the young? Diabetes Care 23:1707-1708

14. Turner R, Stratton I, Horton V et al (1997) UKPDS 25: autoantibodies to islet cell cytoplasm and glutamic acid decarboxylase for prediction of insulin requirement in type 2 diabetes. UK Prospective Diabetes Study Group. Lancet 350:1288-1293

15. Pan CY, So WY, Khalid BA et al (2004) Metabolic, immunological and clinical characteristics in newly diagnosed Asian diabetes patients aged 12-40 years. Diabet Med 21:1007-1013

16. Tica V, Hanif MW, Andersson A et al (2003) Frequency of latent autoimmune diabetes in adults in Asian patients diagnosed as type 2 
diabetes in Birmingham, United Kingdom. Ann N Y Acad Sci 1005:356-358

17. Britten AC, Jones K, Torn C et al (2007) Latent autoimmune diabetes in adults in a South Asian population of the U.K. Diabetes Care 30:3088-3090

18. Sahu RP, Aggarwal A, Zaidi G et al (2007) Etiology of early-onset type 2 diabetes in Indians: islet autoimmunity and mutations in hepatocyte nuclear factor 1alpha and mitochondrial gene. J Clin Endocrinol Metab 92:2462-2467

19. WHO (1995) Physical status: the use and interpretation of anthropometry. Report of a WHO Expert Committee. World Health Organ Tech Rep Ser 854:1-452

20. Trinder P (1969) Determination of blood glucose using 4-amino phenazone as oxygen acceptor. J Clin Pathol 22:246

21. Warnick GR, Knopp RH, Fitzpatrick V, Branson L (1990) Estimating low-density lipoprotein cholesterol by the Friedewald equation is adequate for classifying patients on the basis of nationally recommended cutpoints. Clin Chem 36:15-19

22. Moridani MY, Verjee Z, Allen LC (2003) Analytical evaluation of hemoglobin $\mathrm{A}(1 \mathrm{c})$ dual kit assay on Bio-Rad Variant II: an automated HPLC hemoglobin analyzer for the management of diabetic patients. Clin Biochem 36:317-320

23. Bingley PJ, Bonifacio E, Williams AJ, Genovese S, Bottazzo GF, Gale EA (1997) Prediction of IDDM in the general population: strategies based on combinations of autoantibody markers. Diabetes 46:1701-1710

24. Bingley PJ, Bonifacio E, Mueller PW (2003) Diabetes antibody standardization program: first assay proficiency evaluation. Diabetes 52:1128-1136

25. Matthews DR, Hosker JP, Rudenski AS, Naylor BA, Treacher DF, Turner RC (1985) Homeostasis model assessment: insulin resistance and beta-cell function from fasting plasma glucose and insulin concentrations in man. Diabetologia 28:412-419

26. Alberti KG, Zimmet P, Shaw J (2006) Metabolic syndrome-a new world-wide definition. A consensus statement from the International Diabetes Federation. Diabet Med 23:469-480

27. Hermitte L, Atlan-Gepner C, Mattei C et al (1998) Diverging evolution of anti-GAD and anti-IA-2 antibodies in long-standing diabetes mellitus as a function of age at onset: no association with complications. Diabet Med 15:586-591

28. Borg H, Gottsater A, Fernlund P, Sundkvist G (2002) A 12-year prospective study of the relationship between islet antibodies and beta-cell function at and after the diagnosis in patients with adultonset diabetes. Diabetes 51:1754-1762

29. Desai M, Cull CA, Horton VA et al (2007) GAD autoantibodies and epitope reactivities persist after diagnosis in latent autoimmune diabetes in adults but do not predict disease progression: UKPDS 77. Diabetologia 50:2052-2060

30. Kanungo A, Sanjeevi CB (2003) IA-2 autoantibodies are predominant in latent autoimmune diabetes in adults patients from eastern India. Ann N Y Acad Sci 1005:390-394

31. Tuomi T, Carlsson A, Li H et al (1999) Clinical and genetic characteristics of type 2 diabetes with and without GAD antibodies. Diabetes 48:150-157

32. Thai AC, Ng WY, Loke KY, Lee WR, Lui KF, Cheah JS (1997) Anti-GAD antibodies in Chinese patients with youth and adultonset IDDM and NIDDM. Diabetologia 40:1425-1430

33. Maioli M, Alejandro E, Tonolo G et al (2004) Epitope-restricted 65-kilodalton glutamic acid decarboxylase autoantibodies among new-onset Sardinian type 2 diabetes patients define phenotypes of autoimmune diabetes. J Clin Endocrinol Metab 89:5675-5682

34. Zinman B, Kahn SE, Haffner SM, O, Neill MC, Heise MA, Freed MI (2004) Phenotypic characteristics of GAD antibody-positive recently diagnosed patients with type 2 diabetes in North America and Europe. Diabetes 53:3193-3200

35. Barnett AH, Dixon AN, Bellary S et al (2006) Type 2 diabetes and cardiovascular risk in the UK south Asian community. Diabetologia 49:2234-2246

36. Odugbesan O, Fletcher J, Mijovic C, Mackay E, Bradwell AR, Barnett AH (1987) The HLA-D associations of type 1 (insulindependent) diabetes in Punjabi Asians in the United Kingdom. Diabetologia 30:618-621

37. Rosario PW, Reis JS, Amim R et al (2005) Comparison of clinical and laboratory characteristics between adult-onset type 1 diabetes and latent autoimmune diabetes in adults. Diabetes Care 28:18031804

38. Kyvik KO, Nystrom L, Gorus F et al (2004) The epidemiology of type 1 diabetes mellitus is not the same in young adults as in children. Diabetologia 47:377-384

39. Singh AK, Bhatia E, Dabadghao P, Bhatia V, Gellert SA, Colman PG (2000) Role of islet autoimmunity in the aetiology of different clinical subtypes of diabetes mellitus in young north Indians. Diabet Med 17:275-280

40. Panz VR, Kalk WJ, Zouvanis M, Joffe BI (2000) Distribution of autoantibodies to glutamic acid decarboxylase across the spectrum of diabetes mellitus seen in South Africa. Diabet Med 17:524-527

41. Ohta M, Obayashi H, Takahashi K et al (1996) Radioimmunoprecipitation assay for glutamic acid decarboxylase antibodies evaluated clinically with sera from patients with insulin-dependent diabetes mellitus. Clin Chem 42:1975-1978

42. Vandewalle CL, Falorni A, Svanholm S, Lernmark A, Pipeleers DG, Gorus FK (1995) High diagnostic sensitivity of glutamate decarboxylase autoantibodies in insulin-dependent diabetes mellitus with clinical onset between age 20 and 40 years. The Belgian Diabetes Registry. J Clin Endocrinol Metab 80:846-851

43. Blohme G, Nystrom L, Arnqvist HJ et al (1992) Male predominance of type 1 (insulin-dependent) diabetes mellitus in young adults: results from a 5-year prospective nationwide study of the 15-34-year age group in Sweden. Diabetologia 35:56-62

44. Gale EA, Gillespie KM (2001) Diabetes and gender. Diabetologia 44:3-15

45. Goswami R, Kochupillai N, Gupta N, Kukreja A, Lan M, Maclaren NK (2001) Islet cell autoimmunity in youth onset diabetes mellitus in Northern India. Diabetes Res Clin Pract 53:47-54

46. Zargar AH, Bhat MH, Laway BA, Masoodi SR (2001) Clinical and aetiological profile of early onset diabetes mellitus: data from a tertiary care centre in the Indian subcontinent. J Postgrad Med 47:27-29

47. Gale EA (2005) Latent autoimmune diabetes in adults: a guide for the perplexed. Diabetologia 48:2195-2199

48. Leslie RD, Williams R, Pozzilli P (2006) Clinical review: type 1 diabetes and latent autoimmune diabetes in adults: one end of the rainbow. J Clin Endocrinol Metab 91:1654-1659

49. Cervin C, Lyssenko V, Bakhtadze E et al (2008) Genetic similarities between latent autoimmune diabetes in adults, type 1 diabetes, and type 2 diabetes. Diabetes 57:1433-1437

50. Alberti KG, Zimmet P, Shaw J (2007) International Diabetes Federation: a consensus on type 2 diabetes prevention. Diabet Med 24:451-463 